KIAS-P03014

cond-mat/0302282

\title{
Non-commutative field theory approach to two-dimensional vortex liquid system
}

\author{
Kyungsun Moon ${ }^{1}$, Vincent Pasquier ${ }^{2}$, Chaiho Rim ${ }^{3}$, and Joonhyun Yeo ${ }^{4}$ \\ ${ }^{1}$ Institute of Physics and Applied Physics, Yonsei University \\ Seoul 120-749, Korea \\ kmoon@phya.yonsei.ac.kr \\ 2 Service de Physique Theorique CEA/Saclay \\ Orme des Merisiers F-91191 Gif-sur-Yvette Cedex, FRANCE \\ pasquier@spht.saclay.cea.fr \\ ${ }^{3}$ Department of Physics, Chonbuk National University \\ Chonju 561-756, Korea \\ rim@mail.chonbuk.ac.kr \\ ${ }^{4}$ Department of Physics, Konkuk University \\ Seoul 143-701, Korea \\ jhyeo@konkuk.ac.kr
}

\begin{abstract}
We investigate the non-commutative $(\mathrm{NC})$ field theory approach to the vortex liquid system restricted to the lowest Landau level (LLL) approximation. NC field theory effectively takes care of the phase space reduction of the LLL physics in a $\star$-product form and introduces a new gauge invariant form of a quartic potential of the order parameter in the Ginzburg-Landau (GL) free energy. This new quartic interaction coupling term has a non-trivial equivalence relation with that obtained by Brézin, Nelson and Thiaville in the usual GL framework. The consequence of the equivalence is discussed.
\end{abstract}


1. Introduction: In the presence of a strong magnetic field, condensed matter systems of charged particles are often characterized by the lowest Landau level (LLL) physics. In high-Tc superconductors, for example, thermal fluctuations are much more effective than they are in conventional low-Tc superconductors because of strong anisotropy, high temperature, and short coherence length [1. A large portion of the field-temperature phase diagram of a high-Tc superconductor is occupied by the vortex liquid phase resulting from the melting of the Abrikosov vortex lattice. Since, only fluctuations in the LLL order parameter are important near $H_{c 2}(T)$, the LLL approximation, where the higher Landau modes are neglected all together, has been widely used to study the vortex liquid phase in high-Tc superconductors as well. The higher Landau modes effectively renormalize the phenomenological parameters in the LLL theory, and the quantitative studies [2] of this effect show that the LLL approximation is valid over a wide range of phase diagram below $H_{c 2}(T)$.

The vortex system was orignally studied by Abrikosov [3] using LLL Ginzburg-Landau (GL) theory. Brézin, Nelson and Thiaville (BNT) [4] first studied the fluctuation effects near the upper critical field $H_{c 2}(T)$ in type-II superconductors using the functional renormalization group (RG) on the LLL GL theory and found that the fluctuations drive the phase transition into first order.

On the other hand, the projection to the LLL completely quenches the kinetic energy for the two-dimensional system and induces non-commutativity between two otherwise independent coordinate variables just as it appears in matrix multiplications [5]. Due to this non-commuting nature between coordinates special care has to be taken to analyze LLL.

A useful tool to deal with these systems is the non-commutative (NC) field theory [6, 7], which easily incorporates the phase space reduction of the system. The main advantage of $\mathrm{NC}$ field theory is that one can use the ordinary field theoretical technique used for commuting coordinates, but with field multiplication replaced by the $\star$-product. In fact, NC field appears in many different contexts from quantum gravity at plank scale and string theory [8] to condensed matter system. Recent studies of $\mathrm{NC}$ real scalar field theories show many interesting properties such as non-commutative solitons [9] and phase structure [10].

The quantum Hall $(\mathrm{QH})$ system is considered as the most exemplary case of $\mathrm{NC}$ field theory applicable in condensed matter physics. NC field approach is done for the system with strong magnetic field in [11. The rigid fluid motion is described in terms of NC Chern-Simons field theory to understand quantization of the filling factor of the $\mathrm{QH}$ system [12] and the relation between $\mathrm{NC} U(1)$ gauge theory and fluid mechanics is investigated in [13. In addition, the skyrmion excitations in QH system [14 have been studied based on the NC nature of fermionic variables [15, 16]. In this paper we will consider the vortex liquid system near the upper critical field $H_{c 2}$ in a high-Tc superconductor in a magnetic field. This vortex system is another example of LLL physics, which can be explored using the NC field theory.

The two-dimensional superconductor in a uniform magnetic field is effectively described in terms of GL free energy,

$$
F\left[\Psi, \Psi^{\dagger}\right]=\int d^{2} x\left(\frac{1}{2 m}\left|\left(-i \hbar \vec{\nabla}-\frac{e^{*}}{c} \vec{A}\right) \Psi\right|^{2}+\alpha|\Psi(\vec{x})|^{2}+\frac{\beta}{2}|\Psi(\vec{x})|^{4}\right),
$$


where $\Psi$ is an order parameter representing Cooper pair condensate wave-function and $\alpha, \beta$ and $m$ are phenomenological parameters, and $e^{*}=2 e$. The vector potential is given as $\vec{A}=B(-y / 2, x / 2)$ in the symmetric gauge. We consider the case where the order parameter and its fluctuations are restricted to the LLL. This is believed to be a good approximation over a wide range of the phase diagram below the upper critical field.

The LLL GL free energy [4] is put as $H=H_{2}+H_{4}$ with

$$
H_{2}=\alpha_{2} \int d^{2} x\left|\Psi_{\mathrm{LLL}}(\vec{x})\right|^{2}, \quad H_{4}=\frac{\alpha_{4}}{2} \int d^{2} x\left|\Psi_{\mathrm{LLL}}(\vec{x})\right|^{4}
$$

where $\alpha_{2}=\alpha+\hbar \omega_{c} / 2, \alpha_{4}=\beta$, and $\omega_{c}=e^{*} B /(m c)$ is the cyclotron frequency. The magnetic length $\sqrt{\hbar c / e^{*} B}, \hbar$ and $c$ will be set to 1 in the following. The LLL order parameters are given as $\Psi_{\mathrm{LLL}}(\zeta, \bar{\zeta})=\phi(\zeta) e^{-\zeta \bar{\zeta} / 2}$ where $\phi(\zeta)$ is an arbitrary holomorphic function and $\zeta=(x+i y) / \sqrt{2}(\bar{\zeta}=(x-i y) / \sqrt{2})$ is the holomorphic coordinates (antiholomorphic ones). We will omit the subscript LLL from now on.

For a systematic evaluation of the partition function, the order parameter is conveniently written in the momentum space as

$$
\Psi(\zeta, \bar{\zeta})=\phi(\zeta) e^{-\frac{1}{2} \zeta \bar{\zeta}}=\int \frac{d^{2} k}{(2 \pi)^{2}} \widetilde{\Psi}(k, \bar{k}) \exp \left(\frac{i}{2}(k \bar{\zeta}+\bar{k} \zeta)\right)
$$

where $\widetilde{\Psi}(k, \bar{k})=2 \pi \exp (-k \bar{k} / 2) \phi\left(\frac{2}{i} \frac{\partial}{\partial k}\right)$. After integration by parts one can obtain an equivalent form $\widetilde{\Psi}(k, \bar{k})=2 \pi \exp \left(-\frac{1}{2} k \bar{k}\right) \phi(-i k)$ and $\phi(-i k)$ is the coordinate holomorphic function $\phi(\zeta)$ with $\zeta$ replaced by $-i k$.

As first noted by BNT [4, the renormalization in the LLL can be summarized in an effective gauge invariant quartic term as

$$
H_{4}^{\mathrm{BNT}}=\alpha_{4} \int d^{2} \zeta_{1} d^{2} \zeta_{2}\left|\Psi\left(\zeta_{1}, \bar{\zeta}_{1}\right)\right|^{2} g^{\mathrm{BNT}}\left(\zeta_{1}-\zeta_{2}, \bar{\zeta}_{1}-\bar{\zeta}_{2}\right)\left|\Psi\left(\zeta_{2}, \bar{\zeta}_{2}\right)\right|^{2}
$$

The Fourier transform representation of the quartic term is given as

$$
\begin{aligned}
H_{4}^{\mathrm{BNT}}=\alpha_{4} \int\left[\prod_{i=1}^{4} \frac{d^{2} p_{i}}{(2 \pi)^{2}}\right](2 \pi)^{2} \delta^{(2)}\left(\vec{p}_{1}+\vec{p}_{2}-\vec{p}_{3}-\vec{p}_{4}\right) \tilde{g}^{\mathrm{BNT}}\left(p_{3}-p_{1}, \bar{p}_{3}-\bar{p}_{1}\right) \\
\times(2 \pi)^{4} e^{-\frac{1}{2} \sum_{i} p_{i} \bar{p}_{i}} \phi\left(-i p_{1}\right) \phi\left(-i p_{2}\right) \phi^{\dagger}\left(i p_{3}\right) \phi^{\dagger}\left(i p_{4}\right) .
\end{aligned}
$$

Here $\tilde{g}^{\mathrm{BNT}}(k, \bar{k})$ is the Fourier transform of $g^{\mathrm{BNT}}(\zeta, \bar{\zeta})$,

$$
g^{\mathrm{BNT}}(\zeta, \bar{\zeta})=\int \frac{d^{2} \vec{k}}{(2 \pi)^{2}} \widetilde{g}^{\mathrm{BNT}}(k, \bar{k}) \exp \left(\frac{i}{2}(k \bar{\zeta}+\bar{k} \zeta)\right)
$$

$\tilde{g}^{\mathrm{BNT}}(k, \bar{k})$ takes into account the renormalization of the quartic term starting from the bare value $\tilde{g}_{0}^{\mathrm{BNT}}(k, \bar{k})=1$. As was demonstrated in [17] the Fourier transformed representation of the kernel $g^{\mathrm{BNT}}(\zeta, \bar{\zeta})$ has many advantages in perturbative calculation. The Fourier transform is also directly related to physical quantities describing the vortex liquid such as the structure factor [18]. 
2. NC effective theory: The effective the LLL theory can be reformulated using NC complex bosonic field theory, using the coherent state representation,

$$
\langle\zeta \mid l\rangle=\frac{1}{\sqrt{2 \pi l !}} \zeta^{l} \exp \left(-\frac{1}{2} \bar{\zeta} \zeta\right)
$$

where $|l\rangle$ is the angular momentum state. The coherent states consist of the (over-) complete set of the LLL system,

$$
<\zeta\left|\zeta^{\prime}>=\sum_{l}<\zeta\right| l><l \mid \zeta^{\prime}>=\frac{1}{2 \pi} e^{-\frac{\zeta \bar{\zeta}}{2}-\frac{\zeta^{\prime} \bar{\zeta}^{\prime}}{2}+\zeta \bar{\zeta}^{\prime}} .
$$

The above innocent looking description of the LLL in terms of the coherent states shows an essential feature of the LLL. Note that the coherent state description of the onedimensional harmonic oscillator system comes from the minimal uncertainty wave packet, between the coordinate $x$ and the momentum $p_{x}$. In the LLL, it is not the coordinate and the momentum that do not commute, but the two coordinates $x$ and $y$. The noncommuting property of the two-dimensional coordinates $x$ and $y$, or $\zeta$ and $\bar{\zeta}$ is main result of the phase space reduction due to the strong magnetic field.

Therefore, the two coordinates $\zeta$ and $\bar{\zeta}$ are to be treated as non-commuting operators. This raises the ordering problem of coordinates and results in the awkward calculus of the analysis [5]. To circumvent this inconvenient manipulation, one may introduce the noncommuting operators and the $\star$-product of the corresponding functions 8 . We regard $x$ and $y$ as ordinary commuting coordinates but instead encode the ordering information into the wave-function utilizing the $\star$-product.

Suppose a function $f(\zeta, \bar{\zeta})$ is given as a coherent state expectation value of an operator $O_{f}: f(\zeta, \bar{\zeta})=\left\langle\zeta\left|O_{f}\right| \zeta>\right.$, then $\star$-product of the two functions are defined as the Moyal product

$$
f \star g(\zeta, \bar{\zeta})=\left.\exp \left(\partial_{\bar{\zeta}} \partial_{\bar{\zeta}^{\prime}}-\partial_{\zeta} \partial_{\bar{\zeta}^{\prime}}\right) f(\zeta, \bar{\zeta}) g\left(\zeta^{\prime}, \bar{\zeta}^{\prime}\right)\right|_{\zeta=\zeta^{\prime}}
$$

in consistent with operator product representation,

$$
<\zeta\left|\hat{O}_{f} \hat{O}_{g}\right| \zeta>\equiv<\zeta\left|\hat{O}_{f \star g}\right| \zeta>\text {. }
$$

The hatted operator $\hat{O}_{f}$ can be different from the unhatted one $O_{f}$ by the amount of the normal ordering.

The quadratic term GL free energy is reformulated as

$$
K_{2}=2 \alpha_{2} \int d^{2} \zeta\left\langle\zeta\left|\widehat{\Psi}_{o p}^{\dagger} \widehat{\Psi}_{o p}\right| \zeta\right\rangle=2 \alpha_{2} \int d^{2} \zeta \Psi^{\dagger} \star \Psi(\zeta, \bar{\zeta})=2 \alpha_{2} \int d^{2} \zeta|\Psi(\zeta, \bar{\zeta})|^{2} .
$$

This quadractic part of the two Hamiltonians $H_{2}$ and $K_{2}$ are made equal thanks to the hatted operator and hence by the nature of the Moyal product: Integration of Moyal product of two functions is the same as that of the ordinary product.

The NC quartic term is written as

$$
\begin{aligned}
K_{4}^{\mathrm{NC}}= & \alpha_{4} \int d^{2} \vec{\zeta} d^{2} \vec{\zeta}^{\prime}\left[\Psi \star \Psi^{\dagger}(\zeta, \bar{\zeta})\right] g^{\mathrm{NC}}\left(\zeta-\zeta^{\prime}, \bar{\zeta}-\bar{\zeta}^{\prime}\right)\left[\Psi \star \Psi^{\dagger}\left(\zeta^{\prime}, \bar{\zeta}^{\prime}\right)\right] \\
= & \alpha_{4} \int\left[\prod_{i=1}^{4} \frac{d^{2} p_{i}}{(2 \pi)^{2}}\right](2 \pi)^{2} \delta^{(2)}\left(\vec{p}_{1}+\vec{p}_{2}-\vec{p}_{3}-\vec{p}_{4}\right) \tilde{g}^{\mathrm{NC}}\left(p_{3}-p_{1}, \bar{p}_{3}-\bar{p}_{1}\right) \\
& \times v\left(\left\{p_{i}, \bar{p}_{i}\right\}\right)(2 \pi)^{4} e^{-\frac{1}{2} \sum_{i} p_{i} \bar{p}_{i}} \phi\left(-i p_{1}\right) \phi\left(-i p_{2}\right) \phi^{\dagger}\left(i p_{3}\right) \phi^{\dagger}\left(i p_{4}\right),
\end{aligned}
$$


where the Fourier transformed $\tilde{g}^{\mathrm{NC}}(k, \bar{k})$ is the renormalized one with the bare value $\tilde{g}_{0}^{\mathrm{NC}}(k, \bar{k})=1$. This quartic term includes a new phase factor $v\left(\left\{p_{i}, \bar{p}_{i}\right\}\right)$,

$$
\begin{aligned}
v\left(\left\{p_{i}, \bar{p}_{i}\right\}\right) & =e^{\frac{1}{8}\left[\left(p_{1} \bar{p}_{3}-\bar{p}_{1} p_{3}\right)+\left(p_{2} \bar{p}_{4}-\bar{p}_{2} \bar{p}_{4}\right)\right]}+\left(p_{3} \leftrightarrow p_{4}\right) \\
& =e^{\frac{i}{4}\left[-\left(\vec{p}_{1} \times \vec{p}_{3}\right)-\left(\vec{p}_{2} \times \vec{p}_{4}\right)\right]}+\left(\vec{p}_{3} \leftrightarrow \vec{p}_{4}\right)
\end{aligned}
$$

The quartic term (10) is manifestly different from BNT construction (4), even though both of them are gauge invariant. The gauge transformation is represented as a translation in the vector potential, $\vec{A}(\vec{r}) \rightarrow \vec{A}\left(\vec{r}+\vec{r}_{0}\right)=\vec{A}(\vec{r})+\frac{1}{e^{*}} \vec{\nabla} \chi$ with $\chi(\vec{r})=\left(e^{*} B / 2\right)\left(\vec{r}_{0} \times \vec{r}\right)$ for arbitrary $\vec{r}_{0}$, and the wavefunction transformation

$$
\Psi(\vec{r}) \rightarrow \Psi\left(\vec{r}-\vec{r}_{0}\right) e^{i \frac{e^{*} B}{2}\left(\vec{r}_{0} \times \vec{r}\right)} .
$$

In terms of the holomorphic coordinates, the transformation reads for arbitrary $\zeta_{0}$

$$
\Psi(\zeta, \bar{\zeta}) \rightarrow \Psi\left(\zeta-\zeta_{0}, \bar{\zeta}-\bar{\zeta}_{0}\right) \exp \left[\frac{1}{2}\left(\zeta \bar{\zeta}_{0}-\bar{\zeta} \zeta_{0}\right)\right]
$$

or in the Fourier transformed space

$$
\tilde{\Psi}(k, \bar{k}) \rightarrow \tilde{\Psi}\left(k-k_{0}, \bar{k}-\bar{k}_{0}\right) \exp \left[\frac{1}{2}\left(k \bar{k}_{0}-\bar{k} k_{0}\right)\right]
$$

for arbitrary complex momentum $k_{0}$. The newly introduced phase factor $v\left(\left\{p_{i}, \bar{p}_{i}\right\}\right)$ in $\mathrm{NC}$ quartic term (10) is invariant under the gauge transformation $p \rightarrow p+p_{0}$,

$$
\begin{aligned}
& \left(p_{1} \bar{p}_{3}-\bar{p}_{1} p_{3}\right)+\left(p_{2} \bar{p}_{4}-\bar{p}_{2} p_{4}\right) \\
& \rightarrow\left(p_{1} \bar{p}_{3}-\bar{p}_{1} p_{3}\right)+\left(p_{2} \bar{p}_{4}-\bar{p}_{2} p_{4}\right)+p_{0}\left(\bar{p}_{1}+\bar{p}_{2}-\bar{p}_{3}-\bar{p}_{4}\right)-\bar{p}_{0}\left(p_{1}+p_{2}-p_{3}-p_{4}\right),
\end{aligned}
$$

since the extra contribution proportional to $p_{0}$ ( and to $\bar{p}_{0}$ ) vanishes due to the momentum conservation.

There is an other possibility of the quartic term, $|\Psi \star \Psi|^{2}$. Indeed, the renormalizability of the theory with two terms are investigated in [19]. However, we ruled out the term $|\Psi \star \Psi|^{2}$ because this term is not invariant under the wavefunction transformation (12). Then, there arises a question: how much will the gauge invariant phase factor $v\left(\left\{p_{i}, \bar{p}_{i}\right\}\right)$ affect the correlation. This issue will be answered in the next section.

3. Equivalence relation: The effect of thermal fluctuations in the vortex liquid system is studied by the partition function

$$
Z=\int \mathcal{D} \phi \mathcal{D} \phi^{\dagger} e^{-H\left[\phi, \phi^{\dagger}\right] / k_{B} T}
$$

One can perform the perturbative calculation using the bare propagator obtained from the quadratic term of the Hamiltonian $\mathrm{H}_{2}=K_{2}$

$$
\left\langle\phi^{\dagger}\left(i \bar{k}_{1}\right) \phi\left(-i k_{2}\right)\right\rangle_{0}=\frac{1}{2 \pi \alpha_{2}} e^{\bar{k}_{1} k_{2}}
$$


The $2 n$-point function $G\left(k_{1} \cdots k_{n} ; k_{n+1} \cdots k_{2 n}\right)=\left\langle\phi^{\dagger}\left(i \bar{k}_{1}\right) \cdots \phi^{\dagger}\left(i \bar{k}_{n}\right) \phi\left(-i k_{n+1}\right) \cdots \phi\left(-i k_{2 n}\right)\right\rangle$ and its higher order corrections are evaluated once the lowest order of the 4-point function is known. From $K_{4}$ (10) the four-point function at the tree level is given as

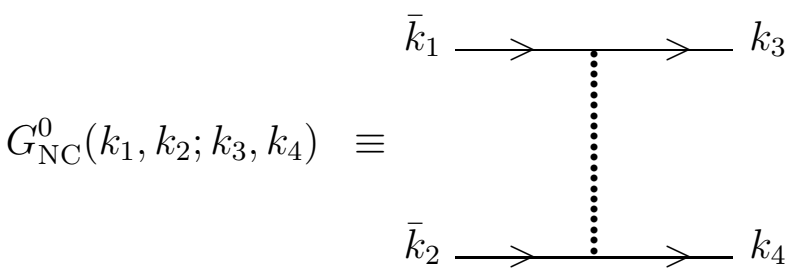

$$
\begin{aligned}
& =-\frac{\alpha_{4}}{\alpha_{2}^{4}}\left(\frac{1}{8 \pi^{2}}\right) \exp \left[\bar{k}_{1} k_{3}+\bar{k}_{2} k_{4}\right] \int \frac{d^{2} p}{(2 \pi)^{2}} \tilde{g}_{0}^{\mathrm{NC}}(p, \bar{p}) \\
& \times \exp \left[-\frac{17}{32} p \bar{p}+\frac{5}{8} \bar{p}\left(k_{3}-k_{4}\right)-\frac{5}{8} p\left(\bar{k}_{1}-\bar{k}_{2}\right)\right] .
\end{aligned}
$$

Rewriting this using $\tilde{f}^{\mathrm{NC}}(\vec{k}) \equiv \exp \left[-\frac{9}{64} k \bar{k}\right] \tilde{g}^{\mathrm{NC}}(\vec{k})$ we have

$$
\begin{aligned}
G_{\mathrm{NC}}^{0}\left(k_{1}, k_{2} ; k_{3}, k_{4}\right)=- & \frac{\alpha_{4}}{\alpha_{2}^{4}}\left(\frac{1}{8 \pi^{2}}\right) \exp \left[\bar{k}_{1} k_{3}+\bar{k}_{2} k_{4}\right] \int \frac{d^{2} p}{(2 \pi)^{2}} \tilde{f}_{0}^{\mathrm{NC}}(p, \bar{p}) \\
& \times \exp \left[-\frac{25}{64} p \bar{p}+\frac{5}{8} \bar{p}\left(k_{3}-k_{4}\right)-\frac{5}{8} p\left(\bar{k}_{1}-\bar{k}_{2}\right)\right] .
\end{aligned}
$$

This is compared with the BNT case;

$$
\begin{aligned}
G_{\mathrm{BNT}}^{0}\left(k_{1}, k_{2} ; k_{3}, k_{4}\right)= & -\frac{\alpha_{4}}{\alpha_{2}^{4}}\left(\frac{1}{8 \pi^{2}}\right) \exp \left[\bar{k}_{1} k_{3}+\bar{k}_{2} k_{4}\right] \int \frac{d^{2} p}{(2 \pi)^{2}} \tilde{g}_{0}^{\mathrm{BNT}}(p, \bar{p}) \\
& \times \exp \left[-\frac{p \bar{p}}{2}+\frac{1}{2} \bar{p}\left(k_{3}-k_{4}\right)-\frac{1}{2} p\left(\bar{k}_{1}-\bar{k}_{2}\right)\right] \\
= & -\frac{\alpha_{4}}{\alpha_{2}^{4}}\left(\frac{1}{8 \pi^{2}}\right) \exp \left[\bar{k}_{1} k_{3}+\bar{k}_{2} k_{4}\right] \int \frac{d^{2} p}{(2 \pi)^{2}} \tilde{f}_{0}^{\mathrm{BNT}}(p, \bar{p}) \\
& \times \exp \left[-\frac{p \bar{p}}{4}+\frac{1}{2} \bar{p}\left(k_{3}-k_{4}\right)-\frac{1}{2} p\left(\bar{k}_{1}-\bar{k}_{2}\right)\right] .
\end{aligned}
$$

where $\tilde{f}^{\mathrm{BNT}} \equiv \exp \left[-\frac{1}{4} k \bar{k}\right] \tilde{g}^{\mathrm{BNT}}(\vec{k})$. Comparing (15) with (17) we have an equivalence relation if we put

$$
\widetilde{f}^{\mathrm{BNT}}(k, \bar{k})=\left(\frac{16}{25}\right) \widetilde{f}^{\mathrm{NC}}\left(\frac{4}{5} k, \frac{4}{5} \bar{k}\right) .
$$

The same relation holds for all order of the perturbation.

4. Remarks and conclusion: We reformulated the lowest Landau level effective GinzburgLandau theory from the non-commutative field theory point of view. This NC theory naturally incorporates the non-commuting nature of coordinates. As the consequence of the non-local behavior of the system due to the phase space reduction, gauge invariant factor $v\left(\left\{p_{i}, \bar{p}_{i}\right\}\right)$ (11) appears in the quartic interaction.

The appearance of the new gauge invariant factor seems not introduce any new physics since there exists the non-trivial equivalence relation (18) between the coupling of $\mathrm{NC}$ and 
that of BNT. Does this equivalence relation demonstrate the nonrelevance of NC field approach to LLL vortex system? The answer is no. This is the disguise of the benefit of the momentum space description.

Note that NC theory has the bare function $\tilde{g}_{0}^{\mathrm{NC}}(k, \bar{k})=1$ or $\tilde{f}_{0}^{\mathrm{NC}}(k, \bar{k})=e^{-\frac{9}{64} k \bar{k}}$ and the corresponding bare function for BNT theory is given as

$$
\tilde{f}_{0}^{\mathrm{BNT}}(k, \bar{k})=\left(\frac{16}{25}\right) \tilde{f}_{0}^{\mathrm{NC}}\left(\frac{4}{5} k, \frac{4}{5} \bar{k}\right)=\frac{16}{25} \exp \left(-\frac{9}{100} k \bar{k}\right),
$$

or in terms of the $g$ function,

$$
\tilde{g}_{0}^{\mathrm{BNT}}(k, \bar{k})=\frac{16}{25} \exp \left(\frac{4}{25} k \bar{k}\right) .
$$

This effective BNT bare coupling cannot be Fourier transformed to the real space though it can be formally put into a non-local form in the real space. In this sense, the NC theory covers the larger domain of coupling constants in coordinate representation where BNT theory becomes unphysical. The gauge invariant phase factor $v\left(\left\{p_{i}, \bar{p}_{i}\right\}\right)$ coming from the NC field theoretical consideration has a very unexpected role from BNT point of view. We note by passing that the specific scaling factor $5 / 4$ comes from the special form of $v\left(\left\{p_{i}, \bar{p}_{i}\right\}\right)$ due to the Moyal product. In general one may introduce the arbitrary power of $v$ without destroying the gauge invariance and change the scaling factor by the same power.

On the other hand, as far as the RG flow concerns, the runaway picture of $g(\zeta, \bar{\zeta})$ does not change and hence, signals the first order phase transition. This can be seen as follows. BNT theory has the bare function $\tilde{g}_{0}^{\mathrm{BNT}}(k, \bar{k})=1$ or $\tilde{f}_{0}^{\mathrm{BNT}}(k, \bar{k})=e^{-\frac{1}{4} k \bar{k}}$. The corresponding bare function of NC theory is given as

$$
\tilde{f}_{0}^{\mathrm{NC}}(k, \bar{k})=\left(\frac{25}{16}\right) \widetilde{f}_{0}^{\mathrm{BNT}}\left(\frac{5}{4} k, \frac{5}{4} \bar{k}\right)=\frac{25}{16} \exp \left(-\frac{25}{64} k \bar{k}\right)
$$

or in terms of the $g$ function,

$$
\tilde{g}_{0}^{\mathrm{NC}}(k, \bar{k})=\frac{25}{16} \exp \left(-\frac{1}{4} k \bar{k}\right), \quad g_{0}^{\mathrm{NC}}(\zeta, \bar{\zeta})=\frac{25}{16 \pi} \exp (-\zeta \bar{\zeta}) .
$$

One can follow the one-loop RG analysis in [4, 17] using the equivalent form (18) and arrive at the same conclusion since the RG flow shares the same structure but with a different initial condition.

Finally, one may study the vortex lattice formation using the NC formalism. Minimizing the free energy is equivalent to minimizing the Abrikosov ratio [3], which, in the $\mathrm{NC}$ theory, is given by

$$
\beta_{A}^{N C}=\frac{1}{A} \int d^{2} r\left(\Psi \star \Psi^{\dagger}\right)^{2} /\left\{\frac{1}{A} \int d^{2} r|\Psi|^{2}\right\}^{2}
$$

where $A$ is the area of the two-dimensional space. The vortex lattice solution satisfies the periodicity condition,

$$
\left|\Psi\left(\vec{r}+\vec{r}_{I}\right)\right|=|\Psi(\vec{r})|, \quad\left|\Psi\left(\vec{r}+\vec{r}_{I I}\right)\right|=|\Psi(\vec{r})|
$$


with arbitrary periodicity vectors parametrized by $\vec{r}_{I}=\ell^{\prime}(1,0)$ and $\vec{r}_{I I}=\ell^{\prime}(\nu, \eta)$. The flux quantization condition gives the area of the unit cell $\eta \ell^{\prime 2}=2 \pi \ell^{2}$ with the magnetic length $\ell$ set to unity in this paper. In terms of the reciprocal lattice vector $\vec{G}_{m n}$, which satisfies $\vec{G}_{m n} \cdot \vec{r}_{I}=2 \pi m$ and $\vec{G}_{m n} \cdot \vec{r}_{I I}=2 \pi n$, one may put

$$
\beta_{A}^{N C}=\frac{16}{25} \sum_{\vec{G}_{m n}} e^{-\frac{9}{100}|\vec{G}|^{2}} .
$$

The minimum value is achieved for a triangular lattice with $\beta_{A}^{N C}=1.7782$. This is, however, only slightly lower than that for the square lattice, $\beta_{A}^{N C}=1.7789$. In the conventional GL theory, the Abrikosov ratio can be written as

$$
\beta_{A}^{B N T}=\sum_{\vec{G}_{m n}} e^{-\frac{1}{4}|\vec{G}|^{2}}
$$

which gives the well known results, $\beta_{A}^{B N T}=1.1596$ for a traingular lattice and $\beta_{A}^{B N T}=$ 1.1803 for a square lattice. We note that this expression can also be obtained if one rescales the NC result by $G \rightarrow \frac{5}{4} G$. From this, one may conclude that a triangular vortex lattice will also be formed in the NC theory, although the difference in free energy between various vortex lattice structures is small compared to that in the conventional GL theory.

In summary, we have studied the vortex system restricted in the LLL using the new Ginzburg-Landau model inspired by the NC field theory. The quartic term in the new GL model differs from the conventional one by the gauge invariant phase factor. We have shown that the effect of this phase factor is the nontrivial rescaling in the correlation functions for the vortex liquids as well as in the mean field quantities describing the vortex lattice. This is, at first sight, quite puzzling, since one cannot rewrite the quartic term (10) in any simpler way by rescaling the momenta into the form without the phase factor $v$. However, once this phase factor is put into Gaussian integrations to calculate the correlation functions, they produce the nontrivial rescaling as we have found in this article. It will be interesting to study the possibility of the physical quantity describing the vortex liquids, for which the phase factor produces other effects than the rescaling. This is left to future work.

Acknowledgements: This work was supported by grant No. R01-1999-00018 from the interdisciplinary research program of the KOSEF. JY and CR would like to acknowledge the hospitality from the YVRC, CR from KIAS and SPhT, and VP from KIAS.

\section{References}

[1] For a review, see G. Blatter, M. V. Feigel'man, V. B. Geshkenbein, A. I. Larkin, and V. M. Vinokur, Rev. Mod. Phys. 66, 1125 (1994).

[2] Z. Tešanović and A. V. Andreev, Phys. Rev. B 49, 4064 (1994); R. Ikeda, J. Phys. Soc. Jpn. 64, 1683 (1995).

[3] A. A. Abrikosov, Zh. Eskp. Theor. Fiz. 32, 1442 (1957) [Sov. Phys.-JETP bf 5, 1174 (1957)]. 
[4] E. Brézin, D. R. Nelson and A. Thiaville, Phys. Rev. B 31 (1985) 7124.

[5] S. M. Girvin and T. Jach, Phys. Rev. B 29, 5617 (1984); G. V. Dunne, R. Jackiw, and C. A. Trugenberger, Phys. Rev. D 41, 661 (1990); N. Read, Phys. Rev. B 58, 16262 (1998).

[6] A. Connes, M. R. Douglas, A. Schwarz, JHEP 9802 (1998) 003.

[7] N. Seiberg and E. Witten, JHEP 9909, 032 (1999).

[8] See e g. J. Madore, "Noncommutative Geometry for Pedestrians", gr-qc/9906059.

[9] R. Gopakumar, S. Minwalla, and A. Strominger, JHEP 0005,020 (2000).

[10] S. Gubser and S. Sondhi, Nucl. Phys. B605, 395 (2001).

[11] D. Bigatti, L. Susskind, Phys.Rev. D 62, 066004 (2000).

[12] L. Susskind, "The Quantum Hall Fluid and Non-Commutative Chern Simons Theory", hep-th/0101029.

[13] R. Jackiw, "Noncommuting fields and non-Abelian fulids", hep-th/0305027 and references therein.

[14] S. L. Sondhi, A. Karlhede, S.A. Kivelson, and E.H. Lezayi, Phys. Rev. B 47, 16419 (1993); K. Moon, H. Mori, Kun Yang, S.M. Girvin, A.H. MacDonald, L. Zheng, D. Yoshioka, and Shou-Cheng Zhang, Phys. Rev. B 51, 5138 (1995); K. Moon, J. Korean Phys. Soc. 35, S35 (1999).

[15] V. Pasquier, Phys. Lett. B490, 258 (2000); Phys. Lett. B513, 241 (2001).

[16] B. H. Lee, K. S. Moon, and C. Rim, Phys. Rev. D 64, 085014 (2001).

[17] M. A. Moore and T. J. Newman, Phys. Rev. Lett. 75, 533 (1995); T. J. Newman and M. A. Moore, Phys. Rev. B 54 (1996-I) 6661.

[18] J. Yeo and M A. Moore, Phys. Rev. Lett. 76 (1996) 1142; Phys. Rev. B 54 (1996) 4218.

[19] I. Ya. Aref'eva, D. M. Belov and A. S. Koshelov, "A Note on UV/IR for Noncommutative Complex Scalar Field", hep-th/0001215; I. Ya. Aref'eva, D. M. Belov, A. S. Koshelov and O. A. Rytchkov, Phys. Lett. B 487, 357 (2000). 